\title{
A spectroscopic study of the close eclipsing binary HS Herculis ${ }^{\star}$
}

\author{
Ö. Çakırlı ${ }^{1,2}$, C. İbanoğlu ${ }^{1}$, and A. Frasca ${ }^{2}$ \\ 1 Ege University, Science Faculty, Astronomy and Space Science Dept., 35100 Bornova, İzmir, Turkey \\ 2 INAF - Catania Astrophysical Observatory, via S. Sofia 78, 95123 Catania, Italy \\ e-mail: ock@oact.inaf.it
}

Received 11 May 2007 / Accepted 17 August 2007

\begin{abstract}
Aims. New high-resolution spectroscopic observations of the eclipsing binary HS Herculis are presented. The main aims were to determine the stellar parameters of the two components, their evolutionary stage, and to search for spectroscopic evidence for a possible third body.

Methods. Using the cross-correlation technique, we detect spectroscopically the faint secondary component of HS Herculis, and for the firts time measure its radial velocity. The primary and secondary radial velocity curves are analyzed simultaneously and the results of the orbital solution are combined with those derived from multiband light curve analysis to derive orbital and stellar parameters. Results. We find the masses to be $M_{1}=4.49 \pm 0.16$ and $M_{2}=1.75 \pm 0.09 M_{\odot}$, the radii to be $R_{1}=2.83 \pm 0.04$ and $R_{2}=1.61 \pm 0.02 R_{\odot}$, and the effective temperatures to be $T_{1}=15200 \pm 750 \mathrm{~K}$ and $T_{2}=7600 \pm 400 \mathrm{~K}$ for the primary and secondary stars, respectively. We also derive projected rotational velocities of the components as $v_{1} \sin i=81 \pm 3$ and $v_{2} \sin i=24 \pm 6$.

Conclusions. While a synchronous rotation for the primary star is indicated by the broadening of the spectral lines, the secondary component appears to rotate more slowly, nearly one half the synchronous rotation velocity. This discrepancy indicates that the less massive secondary component could have not yet attained tidal synchronization. Although the presence of a third body physically bound to the eclipsing pair has been suggested by many investigators, we find no sign of its presence in our CCD spectra. The evolutionary stage of the system's components is briefly discussed by comparing their physical parameters with those of theoretical models. We find that the two components are located near the zero-age main sequence, with an age of about $32 \mathrm{Myr}$.
\end{abstract}

Key words. techniques: radial velocities - techniques: spectroscopic - stars: binaries: close - stars: binaries: eclipsing stars: individual: HS Herculis

\section{Introduction}

HS Herculis, hereafter HS Her, was discovered as a variable star in 1934 by Martynov (1940), using visual observations spanning from 1934 to 1943. He classified it as an Algol-type eclipsing binary from the inspection of the visual light curve. The variability and binary nature of HS Her were also discovered independently and almost simultaneously by Jacchia (1940) using Harvard plates, which were taken between 1898 and 1939. The first spectroscopic observations made by Cesco \& Sahade (1945) revealed that the spectral lines visible in their spectra originate from the primary (more massive) component. The lines of the secondary component were not detected due to its faintness and the system was classified as a single-lined spectroscopic binary (SB1). They classified the more-massive primary component as $\mathrm{B} 5-8 \mathrm{~V}$ and calculated the mass function as $0.095 M_{\odot}$. The more massive component was subsequently classified as B4V by Hall \& Hubbard (1971), based on multiwavelength wide band photometry, and as B6III by Etzel \& Olson (1993), from the spectra taken at the Mt. Laguna Observatory.

Most of the studies made on HS Her are concentrated on the determination of apsidal motion rate and the presence of a third body dynamically bounded to the eclipsing system. Wolf et al. (2002) confirmed not only an apsidal motion with a period of 78 years but also suggested the presence of a third body orbiting around the center-of-mass of the inner binary in a highly

^ Based on observations collected at Catania Astrophysical Observatory, Italy. eccentric orbit $(e \simeq 0.80)$ with a period of 85.7 years. This result was based on the analysis of the $\mathrm{O}-\mathrm{C}$ residuals, the differences between the observed and calculated times for mideclipses. Çolak \& Müyesseroğlu (2005) collected and analyzed all the times of mid-eclipses available in the literature. They explained the observed $\mathrm{O}-\mathrm{C}$ variation as being due to the apsidal motion with a period of about 77 years, and rejected the third body hypothesis. Very recently, Khaliullin \& Khaliullina (2006) and Bozkurt \& Değirmenci (2006) analyzed all the O-C residuals and concluded that these cannot be represented by an apsidal motion alone. The presence of a third body was suggested once again by both studies. The mass of the third body was estimated to be $1-2 M_{\odot}$ by Khaliullin \& Khaliullina and a minimum mass of $1.64 M_{\odot}$ was suggested by Bozkurt \& Deǧirmenci. While the orbital eccentricity for the outer orbit was estimated to be larger than 0.70 in the former study, an eccentricity of 0.90 was suggested in the latter. Khaliullin \& Khaliullina also discussed the evolutionary status of the components and suggested that the secondary component of the system is a pre-main-sequence star still in the contracting phase towards the zero-age main sequence. Bozkurt \& Değirmenci (2006) combined the parameters found from the solution of their $U B V$ light curves with those obtained by Cesco \& Sahade (1945) from the analysis of the primary's radial velocity and obtained masses of $6 M_{\odot}$ and $1.8 M_{\odot}$ for the primary and secondary components, respectively.

The percentage of triple and multiple star systems is considerably high in our galaxy (e.g. Chambliss 1992; Demircan 2000). The discovery of multiple systems and the determination 
of the physical parameters of their components is of great importance because they are likely related to star formation in environments with high stellar density. We found in the literature no high-resolution spectroscopic study of HS Her and no direct mass determination of both components. This motivated us to perform high-resolution spectroscopic observations of HS Her in order to detect signatures of an eventual third body and to improve the physical and orbital parameters of the system.

\section{Echelle spectroscopy}

Spectroscopic observations were performed with the FRESCO echelle spectrograph at the $91-\mathrm{cm}$ telescope of Catania Astrophysical Observatory. The spectrograph is fed by the telescope through an optical fiber (UV-NIR, $100 \mu \mathrm{m}$ core diameter) and is located in a stable position in the room below the dome level. Spectra were recorded on a CCD camera equipped with a thinned back-illuminated SITe CCD of $1024 \times 1024$ pixels (size $24 \times 24 \mu \mathrm{m})$. The resolution is $\simeq 20000$, as deduced from the full width at half maximum of the lines of the Th-Ar calibration lamp. The spectra cover the wavelength range from 4300 to $6650 \AA$, split into 19 orders. In this spectral region there are several lines useful for measuring radial velocity and for the star classification, mainly located in the blue portion of the spectrum. The data reduction was performed by using the ECHELLE task of IRAF ${ }^{1}$ package following the standard steps: background subtraction, division by a flat field spectrum given by a halogen lamp, wavelength calibration using the emission lines of a Th-Ar lamp, and normalization to the continuum through a polynomial fit.

Nineteen spectra of HS Her were collected during the 22 observing nights between August 2 and 23, 2005. Typical exposure times for the HS Her spectroscopic observations were between 3000 and $3600 \mathrm{~s}$. The signal-to-noise ratio $(\mathrm{S} / \mathrm{N})$ achieved was between 40 and 101, depending on the atmospheric conditions. $\alpha$ Lyr (A0V), 59 Her (A3IV), and $\iota$ Psc (F7V) were observed during each run as radial velocity standard stars. The slowly-rotating star HD 27962 (A2IV) was observed as a template for the measurements of rotational velocity. The average $\mathrm{S} / \mathrm{N}$ at continuum in the spectral region of interest was 170-350 for the standard stars.

\section{Spectral classification}

We have used our spectra to classify the primary component of HS Her. For this purpose we have degraded the spectral resolution from 22000 to 3000 , by convolving them with a Gaussian kernel of the appropriate width, and we have measured the equivalent width $(E W)$ of photospheric absorption lines useful for the spectral classification of hot stars. We have followed the guidelines of Hernández et al. (2004), choosing hydrogen and helium lines in the blue-wavelength region, where the contribution of the secondary component to the observed spectrum is negligible. From several spectra we measured $E W_{\mathrm{H} \gamma}=7.6 \pm 0.6 \AA$, $E W_{\mathrm{HeI} \lambda 4388}=0.48 \pm 0.05 \AA, E W_{\mathrm{HeI} \lambda 4471}=0.79 \pm 0.13 \AA$, and $E W_{\mathrm{HeI} \lambda 4922}=0.51 \pm 0.04 \AA$.

From the calibration relations $E W$-Spectral-Type of Hernández et al. (2004), we have derived a spectral type B4.5V

${ }^{1}$ IRAF is distributed by the National Optical Observatory, which is operated by the Association of the Universities for Research in Astronomy, inc. (AURA) under cooperative agreement with the National Science Foundation.

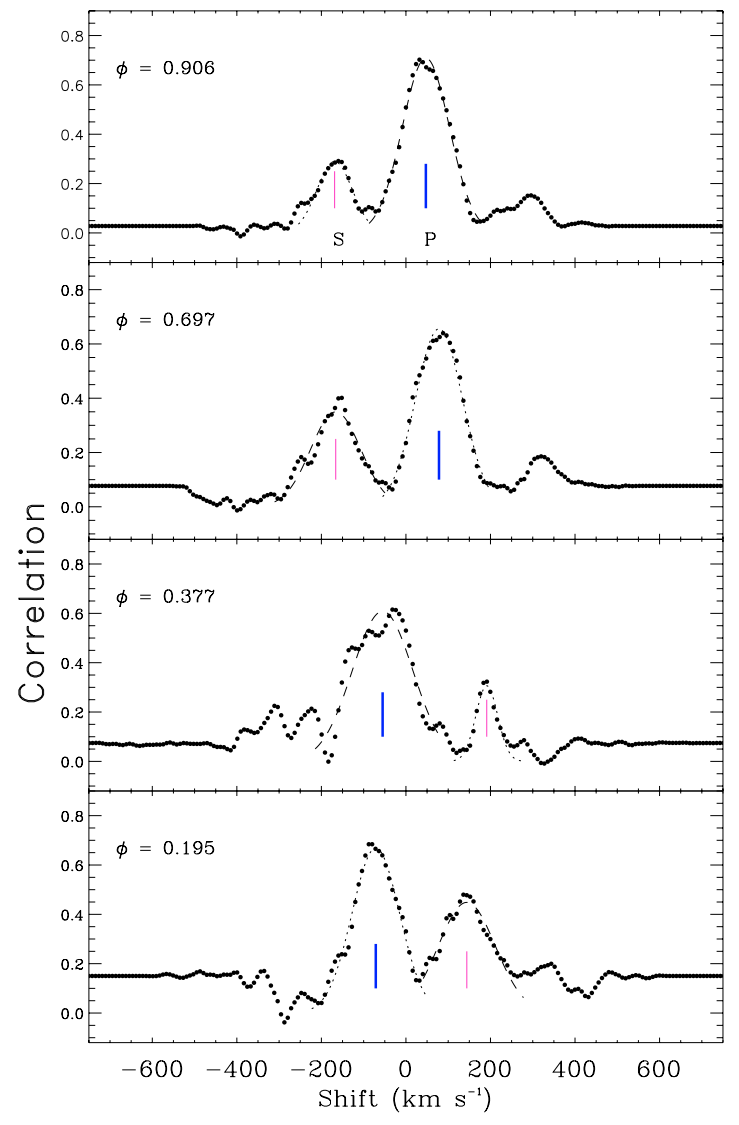

Fig. 1. Sample of cross correlation functions between HS Her and the RV template spectra at four orbital phases near the quadratures.

with an uncertainty of about 0.7 spectral subclass. The effective temperature deduced from the calibrations of Drilling \& Landolt (2000) or de Jager \& Nieuwenhuijzen (1987) is about $16000 \mathrm{~K}$. The spectral-type uncertainty leads to a temperature error $\Delta T_{\mathrm{eff}} \approx 1000 \mathrm{~K}$.

The drawback of this method is the need of a calibration relation Spectral-Type $-T_{\text {eff }}$, but it has the advantage of being practically unaffected by the interstellar reddening, unlike the color indices.

\section{Spectroscopic analysis}

Double-lined spectroscopic binaries are characterized by the presence of two stellar spectra that appears in the crosscorrelation function $(\mathrm{CCF})$ as two peaks displacing back and forth according to the orbital motion of the system's components (Fig. 1). The location of the two peaks allows the measurement of the radial velocity of each component at the phase of observation. This helps to explore the binary mass-ratio distribution, especially in the low-mass regime, where the secondary components are usually faint and therefore hard to detect. The crosscorrelation technique applied to digitized spectra is now one of the standard tools for the measurement of radial velocities in close binary systems. The CCF procedure strongly emphasizes the spectral signatures of relatively faint secondary components in spectroscopic binaries.

The radial velocity measurements of HS Her were obtained by cross-correlation of each echelle order of HS Her's spectra with the spectra of the bright radial velocity standard stars $\alpha$ Lyr (A0V), 59 Her (A3IV) and $\iota$ Psc (F7V), whose radial velocities are $-13.5 \mathrm{~km} \mathrm{~s}^{-1},-12.4 \mathrm{~km} \mathrm{~s}^{-1}$, and $+5.4 \mathrm{~km} \mathrm{~s}^{-1}$, respectively, 
Table 1. Heliocentric radial velocities of HS Her. The columns give the heliocentric Julian date, the orbital phase (according to the ephemeris given by Bozkurt \& Değirmenci 2006), the radial velocities of the two components with the corresponding errors, and the average $\mathrm{S} / \mathrm{N}$ of the spectrum. An " $n$ " means that no reliable measurement could be performed for the secondary, either because of a low $\mathrm{S} / \mathrm{N}$ ratio or due to severe blend between lines of the primary and secondary component.

\begin{tabular}{ccrrrrc}
\hline \hline HJD & Phase & \multicolumn{3}{c}{ Star 1} & \multicolumn{3}{c}{ Star 2} & $\langle S / N\rangle$ \\
$2453000+$ & & $\mathrm{RV}_{\odot}$ & $\sigma$ & $\mathrm{RV}_{\odot}$ & $\sigma$ & \\
\hline 586.4801 & 0.9056 & 38.8 & 5.2 & -162.0 & 12.5 & 74 \\
587.4198 & 0.4795 & -33.0 & 7.0 & $n$ & - & $86^{a}$ \\
588.4303 & 0.0966 & -63.0 & 9.9 & $n$ & - & $<40$ \\
589.4141 & 0.6975 & 65.0 & 3.2 & -208.2 & 27.5 & $75^{a}$ \\
590.4084 & 0.3047 & -110.0 & 4.8 & 213.4 & 14.6 & 65 \\
593.4132 & 0.1398 & -84.0 & 2.6 & 166.9 & 26.8 & 45 \\
594.4441 & 0.7693 & 80.0 & 4.1 & -240.3 & 8.4 & $80^{a}$ \\
595.4386 & 0.3767 & -88.1 & 4.7 & 149.1 & 21.9 & $90^{a}$ \\
596.4243 & 0.9787 & 12.3 & 1.4 & $n$ & - & $90^{a}$ \\
597.3797 & 0.5622 & -4.0 & 1.4 & $n$ & - & $101^{a}$ \\
598.4156 & 0.1948 & -101.4 & 4.4 & 211.7 & 18.8 & $93^{a}$ \\
599.4588 & 0.8319 & 74.1 & 5.3 & -239.3 & 9.9 & 55 \\
600.4637 & 0.4456 & -53.1 & 9.3 & $n$ & - & 67 \\
602.3716 & 0.6108 & 26.0 & 7.0 & $n$ & - & $101^{a}$ \\
604.4569 & 0.8843 & 52.5 & 3.1 & -189.0 & 11.2 & 72 \\
605.4010 & 0.4609 & -42.7 & 5.9 & $n$ & - & 69 \\
\hline
\end{tabular}

Used also for rotational velocities $(v \sin i)$ measurements.

determined by Nordstroem et al. (2004) from new accurate radial velocity observations for nearly 13500 stars. For this purpose the IRAF task fxcor was used. We have also formed the synthetic templates at $15000 \mathrm{~K}$ and $7500 \mathrm{~K}$ spectra covering the wavelength range from 4300 to $6650 \AA$ using Kurucz ATLAS9 models in order to measure radial velocities of the components. The maxima of the CCF, corresponding to the primary and secondary components, are fairly similar to those obtained from the observed ones. Therefore, the measured RVs did not change significantly, but the uncertainties in the RVs of the secondary component are a little bit larger.

Figure 1 shows examples of CCFs of HS Her near the first and second quadrature. The two non-blended peaks correspond to each component of HS Her. We applied the cross-correlation technique to five wavelength regions with well-defined absorption lines of the primary and secondary components. These regions include the following lines: Si III $4568 \AA, \mathrm{Mg}$ II $4481 \AA$, He I $5016 \AA$, He I $4917 \AA$, He I $5876 \AA$. The stronger CCF peak corresponds to the more massive component that also has a larger contribution to the observed spectrum. To better evaluate the centroids of the peaks (i.e., the radial (RV) velocity difference between the target and the template), we adopted two separate Gaussian fits for the case of significant peak separation.

The radial velocity measurements, listed in Table 1 together with their standard errors, are weighted means of the individual values deduced from each order (see, e.g., Frasca et al. 2006). The observational points and their error bars are displayed in Fig. 2 as a function of orbital phase as calculated by means of the ephemeris based on the photometric times of the primary eclipse described in Bozkurt \& Değirmenci (2006).

The first detailed solution of both radial velocity curves of HS Her components is presented in this study. We found the semi-amplitude of the more massive, more luminous component to be $K_{1}=93.4 \pm 2.8 \mathrm{~km} \mathrm{~s}^{-1}$. We could clearly detect the CCF peak of the secondary component in only nine spectra

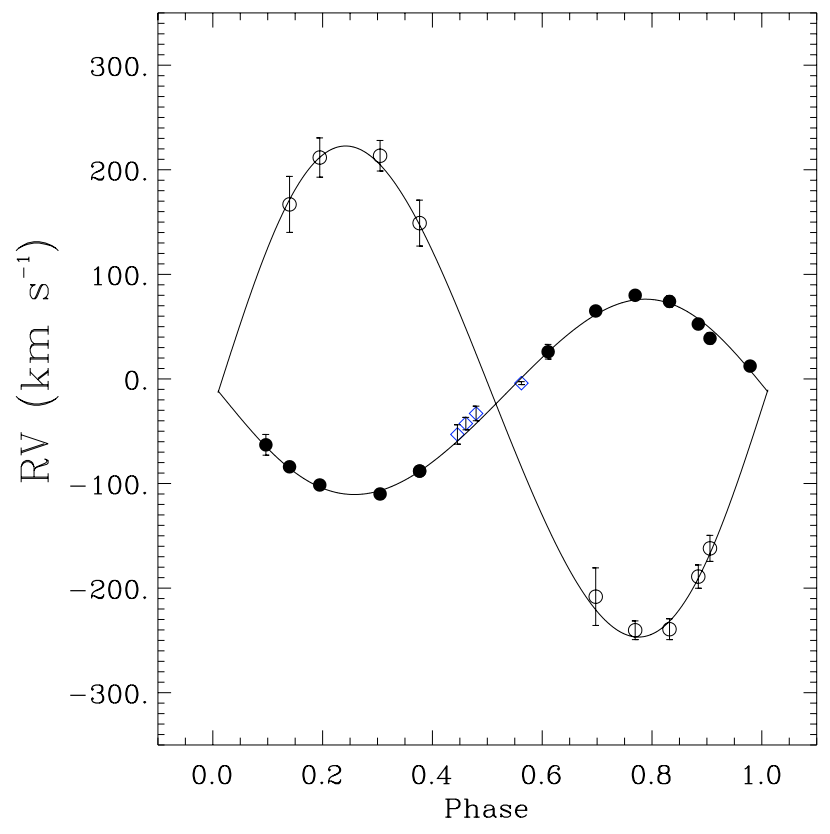

Fig. 2. New radial velocities obtained by us and the corresponding double-lined orbit for HS Her plotted as a function of orbital phase. Open and filled circles represent the velocities of the secondary and primary components, respectively. Error bars are masked by the symbol size in many cases. Open diamonds represent RV data taken near the secondary eclipse. A zero weight has been given to these data in calculating the orbital solution.

acquired far from the conjunctions. From these RV values we found $K_{2}=239.5 \pm 3.2 \mathrm{~km} \mathrm{~s}^{-1}$.

The width of the cross-correlation profile is a good tool for the measurement of $v \sin i$ (see, e.g., Queloz et al. 1998). The rotational velocities $(v \sin i)$ of the two components were obtained by measuring the FWHM of the CCF peaks in nine high-S/N spectra of HS Her acquired close to the quadratures, where the spectral lines have the largest Doppler-shifts. In order to construct a calibration curve FWHM- $v \sin i$, we have used an average spectrum of HD 27962, acquired with the same instrumentation. Since the rotational velocity of HD 27962 is very low but not zero $\left(v \sin i \simeq 11 \mathrm{~km} \mathrm{~s}^{-1}\right.$, e.g., Royer et al. 2004 and references therein), it could be considered as a useful template for A-type stars rotating faster than $v \sin i \simeq 10 \mathrm{~km} \mathrm{~s}^{-1}$. The spectrum of HD 27962 was synthetically broadened by convolution with rotational profiles of increasing $v \sin i$ in steps of $5 \mathrm{~km} \mathrm{~s}^{-1}$ and the cross-correlation with the original one was performed at each step. The FWHM of the CCF peak was measured and the FWHM- $v \sin i$ calibration was established. The $v \sin i$ values of the two components of HS Her were derived from the FWHM of their CCF peak and the aforementioned calibration relations, for a few wavelength regions and for the best spectra. This gave values of $80.8 \pm 3.2 \mathrm{~km} \mathrm{~s}^{-1}$ for the primary star and $24 \pm 6.1 \mathrm{~km} \mathrm{~s}^{-1}$ for the secondary star. The rotation rate of the primary component derived by Etzel \& Olson, $83 \pm 4 \mathrm{~km} \mathrm{~s}^{-1}$, is in very good agreement with our determination.

\section{Physical properties of HS Her}

As previously mentioned, the radial velocity curve of the more massive component obtained and analyzed by Cesco \& Sahade (1945). They gathered 49 RV measurements over 6 months. They detected only the lines of the more massive star and their analysis does not provide the full set of orbital and stellar 
Table 2. Properties of the HS Her components.

\begin{tabular}{lcr}
\hline \hline Parameters & Cesco \& Sahade & This study \\
\hline$P($ days $)$ & 1.637416 & $1.6374316^{a}$ \\
$T_{0}(\mathrm{HJD})$ (Min I) & 2428771.361 & 2447382.4063 \\
$a\left(R_{\odot}\right)$ & - & $10.76 \pm 0.50$ \\
$\gamma\left(\mathrm{km} \mathrm{s}^{-1}\right)$ & $-16.0 \pm 1.1$ & $-12.8 \pm 1.9$ \\
$q=\frac{m_{2}}{m_{1}}$ & - & $0.39 \pm 0.05$ \\
$i$ & - & $88.09^{a}$ \\
$T(\mathrm{HJD})$ (Periastron) & - & 2453584.9525 \\
$\omega($ Periastron Longitude $)$ & 37 & $72 \pm 2$ \\
$K_{1}\left(\mathrm{~km} \mathrm{~s}^{-1}\right)$ & 82.6 & $93.4 \pm 2.8$ \\
$K_{2}\left(\mathrm{~km} \mathrm{~s}^{-1}\right)$ & - & $239.5 \pm 3.2$ \\
$e$ & 0.05 & $0.05 \pm 0.01$ \\
$R_{1}^{b}\left(R_{\odot}\right)$ & - & $2.83 \pm 0.04$ \\
$R_{2}^{b}\left(R_{\odot}\right)$ & - & $1.61 \pm 0.02$ \\
$M_{1}^{b}\left(M_{\odot}\right)$ & - & $4.49 \pm 0.16$ \\
$M_{2}^{b}\left(M_{\odot}\right)$ & - & $1.75 \pm 0.09$ \\
$L_{1}^{b} / L_{\odot}$ & - & $386 \pm 1$ \\
$L_{2}^{b} / L_{\odot}$ & - & $8 \pm 1$ \\
$T_{\text {eff1 }}(\mathrm{K})$ & - & $15200 \pm 750$ \\
$T_{\text {eff2 }}(\mathrm{K})$ & - & $7600 \pm 400$ \\
$\log _{\log } g_{1}^{b}$ & - & $4.19 \pm 0.01$ \\
$\log _{g_{2}^{b}}^{b}$ & - & $4.27 \pm 0.01$ \\
$v_{\text {syn }}^{b} \sin i\left(\mathrm{~km} \mathrm{~s}^{-1}\right)$ & - & $87.47 \pm 1.15$ \\
$v_{\text {syn }}^{b} \sin i\left(\mathrm{~km} \mathrm{~s}^{-1}\right)$ & - & $49.64 \pm 0.65$ \\
$M_{\text {bol }}^{b}$ & - & $-0.62 \pm 0.06$ \\
$M_{\text {bol }}^{b}$ & - & $2.75 \pm 0.15$ \\
$d_{1}^{b}(\mathrm{pc})$ & - & $531 \pm 34$ \\
$d_{2}^{b}(\mathrm{pc})$ & - & $629 \pm 57$ \\
$d^{b}(\mathrm{pc})$ & - & $535 \pm 34$ \\
\hline
\end{tabular}

${ }^{a}$ Adopted from Bozkurt \& Değirmenci (2006).

${ }^{b}$ Output from JKTABSDIM. Calculated assuming $L_{\odot}=3.826 \times 10^{26} \mathrm{~W}$ and $M_{\mathrm{bol}} \odot=4.75$.

parameters. In Fig. 2 we plot the observed radial velocities and their error bars. Our data have much smaller error bars and appear much less scattered compared to those of Cesco \& Sahade. Notwithstanding this scatter, a small offset between the two data sets seems to be present and could be the effect of a third body. Indeed, the value of the systemic velocity $(\gamma)$ found by us is significantly different (taking into account the errors) from that of Cesco \& Sahade (Table 2). This supports the hypothesis that a third body is responsible for the $\mathrm{O}-\mathrm{C}$ variation. Moreover, we find that the semi-amplitude of the RV curve of the primary component is approximately $13 \%$ larger than that estimated by Cesco \& Sahade. We used the JKTABSDIM ${ }^{2}$ code, which calculates distances and other physical parameters using several different sources of bolometric corrections (Soutworth et al. 2005a). The best fitting parameters are listed in Table 2 together with those obtained by Cesco \& Sahade (1945). The corresponding RV curves are shown in Fig. 2.

We determined the temperature of the primary component of HS Her, $T_{1} \simeq 16000 \mathrm{~K}$, from its spectral-type B4.5 (see Sect. 3). However, as a further and independent diagnostic of effective temperature, we have used the published photometry. By searching the $\mathrm{GCPD}^{3}$, we found $U B V$ photometry made by Oja (1991) who report $V=8$. $56, B-V=+0.03$, and $U-B=-0.42$. For the evaluation of the de-reddened colors, we used Johnson's $Q$-method with the equations for the reddening-free parameters, $Q$ and $D$, given by Hovhannessian (2004). With the values

\footnotetext{
2 This can be obtained from

http://www . astro.keele.ac.uk/ jkt/codes.html

3 http://obswww. unige.ch/gcpd/gcpd.html
}

of $U-B$ and $B-V$ reported by Oja (1991) and adopting a value for the ratio of excesses $[E(U-B) / E(B-V)]=0.72 \pm 0.03$, as suggested by Hovhannessian (2004), we found $D=0.313$ and $Q=-0.442$. From the tables of Hovhannessian (2004) a de-reddened color index $(B-V)_{0}=-0.17 \pm 0$. 01 can be read and a temperature of $15200 \pm 700 \mathrm{~K}$ can be derived from the calibration of Drilling \& Landolt (2000). This value is more consistent with a B5V spectral type. We have adopted the value of $T_{1}=15200 \mathrm{~K}$ for the following analysis. The temperature of the secondary component, $T_{2}=7600 \pm 350 \mathrm{~K}$, has been derived from $T_{1}$ and from the temperature ratio provided by the solution of Bozkurt \& Değirmenci (2006). The luminosities of both components of HS Her have also been obtained from the solution of the light curve by Bozkurt \& Değirmenci (2006), which provides the stellar radii in relative units. These were subsequently converted into absolute units through our own determination of the system semi-major axis based on the RV curves. In Fig. 5, we plot luminosity versus effective temperature for both components, i.e., the Hertzsprung-Russell (HR) diagram. For comparison, two isochrones of Siess et al. (2000) at an age of 1.6 Myr (dotted line), corresponding to the pre-main sequence phase, and at $32 \mathrm{Myr}$ (dashed line), have been shown in Fig. 5a. The bestfit for the components of HS Her is at an age of $32 \mathrm{Myr}$ and a metallicity of $Z=0.02$, i.e., both components have already reached the zero-age main sequence (ZAMS). Figure $5 \mathrm{~b}$ displays the mass-radius relation according to the models for $Z=0.02$ and $Z=0.03$ and observed properties of HS Her with error bars. The best-fitting isochrone corresponds to $Z=0.02$.

\section{Third component}

Bastian (1993) was the first to suggest the existence of a tertiary component in HS Her. He rejected the apsidal motion to explain the $\mathrm{O}-\mathrm{C}$ variation and suggested a light-time effect due to a third body orbiting around the close binary, with a period of the order of 60 years. On the contrary, Todoran \& Agerer (1994) interpreted the $\mathrm{O}-\mathrm{C}$ residuals as being due to the apsidal motion and rejected the hypothesis of a third body. Wolf et al. (2002) determined a period of 78 years for the apsidal motion, which was not sufficient to explain the $\mathrm{O}-\mathrm{C}$ behavior and proposed, in addition to the apsidal motion, a light-time effect with a period of about 85 years. In a very recent work, Bozkurt \& Değirmenci (2006) confirmed not only the apsidal motion with a period of 80.7 years but even the presence of a third body, physically bound to the system and orbiting in a highly eccentric orbit $(e=0.90)$ with a period of about 85 years.

If its contribution to the total luminosity is not too small, a tertiary component leaves its signatures in the observed spectrum of a double-lined binary. Additional lines, other than those from the components of the close binary, can be attributed to a third body. CCF analysis now allows the detection of very faint satellite lines, down to a relative luminosity of only $0.5 \%$ in a binary system, if high $\mathrm{S} / \mathrm{N}$ spectra are available (e.g., Vitrichenko et al. 2006). Another way to detect spectroscopically a third body orbiting around a binary system is offered by the variation of its systemic velocity $(\gamma)$. The rate of variation of the systemic velocity depends upon the orbital period of the third body. The longer the period, the smaller the $\gamma$ variation, and vice versa. A well known example of a triple system with a tertiary component discovered by means of this effect is BM Ori; the third body is too faint to display its lines in the observed spectrum (Hall 1971).

We could easily detect the CCF peak of the secondary component in the spectra taken near the quadratures. However, absorption lines, and CCF peaks between those of the two 


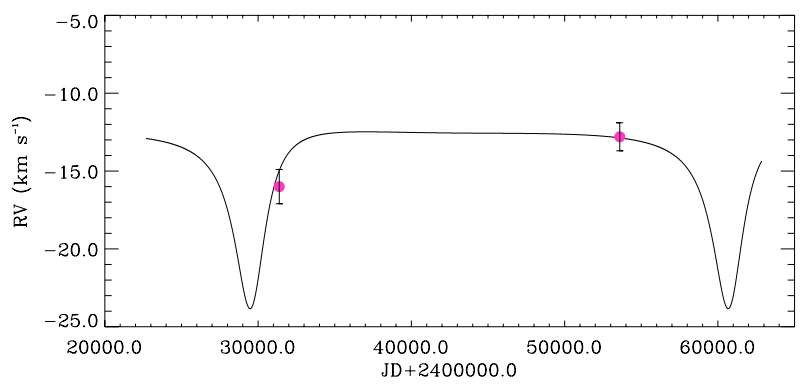

Fig. 3. The systemic radial velocity of HS Her in two observing runs is displayed by filled dots. The orbital solution derived by Bozkurt \& Degirmenci (2006) is shown with a continuous line.

components that could be attributed to a third star were never detected. According to the minimum mass of $1.65 M_{\odot}$ estimated by Bozkurt \& Değirmenci, its spectral lines should be recognized in the best spectra taken near the quadratures of the eclipsing pair. We have updated the value of minimum mass evaluated by Bozkurt \& Değirmenci on the basis of their mass function, $f\left(M_{3}\right)=0.0485 M_{\odot}$, with the total mass of the inner binary found by us, $M_{1}+M_{2}=6.24 M_{\odot}$, finding $M_{3, \min }=1.42 M_{\odot}$. This does not change the previous conclusion about the expected detectability of the third star's spectrum. In any case, we have computed the RV curve expected for the barycenter of the close binary assuming the values of the third-body orbit listed by Bozkurt \& Değirmenci (2006) in their Table 2 and an inclination for the tertiary orbit $i=90^{\circ}$. The expected RV curve of the barycenter of HS Her binary is depicted in Fig. 3 (full line) together with the measured values of $\gamma$ velocity (dots). In this hypothesis, the semi-amplitude of the radial velocity of the eclipsing pair's barycenter is about $6 \mathrm{~km} \mathrm{~s}^{-1}$. The two values of $\gamma$ velocity lie on the theoretical curve, but their position is in the flat portion of the curve and their errors prevent us from confirming the presence of a third body in the HS Her system. This plot also shows that the largest change in the systemic velocity of the eclipsing pair would be around HJD $=2461000$, i.e., near the end of 2025 . It would certainly be very interesting to monitor spectroscopically this system in the future, especially from 2015, when the $\gamma$ velocity should decrease appreciably if the third body hypothesis and orbital solution is correct.

Assuming a minimum mass of $1.42 M_{\odot}$ for this hypothetical third body, we can estimate a semi-major axis for its orbit to be about 38 AU. Therefore, with an eccentricity $e=0.9$ and an inclination of the tertiary orbit close to $90^{\circ}$, the minimum and maximum separations between the eclipsing pair and the third star would be 3.8 and 73 AU, respectively. Using a parallax of $2.01 \pm 1.04$ mas given by the Hipparcos satellite, we can estimate the minimum and maximum angular separations as 0.008 and 0.145 arcsec, respectively. Even the maximum separation is very close to the Hipparcos resolution limit of 0.1 arcsec. This implies that the non-detection of HS Her as a visual binary by Hipparcos does not exclude the possibility of the presence of a third body.

However, the non-detection of spectral lines or CCF peaks compatible with a third body, and the very low third-light $(\leq 2 \%)$ found by light curve solutions (e.g., Bozkurt \& Deǧirmenci 2006), is at odds with the third star $\left(M \geq 1.42 M_{\odot}\right)$ hypothesis. The current detection limit for the spectroscopic companions, given by the ratio of the third component light to the total light of the eclipsing pair $l_{3} /\left(l_{1}+L_{2}\right)$, is about $0.03-0.04$. In the case of HS Her the ratio of 0.02 corresponds to $\Delta m \sim 4.2$, which is below the detection limit of $3.8 \mathrm{mag}$. If the third body is a main sequence star with a mass about $1.4 M_{\odot}$, its contribution to the

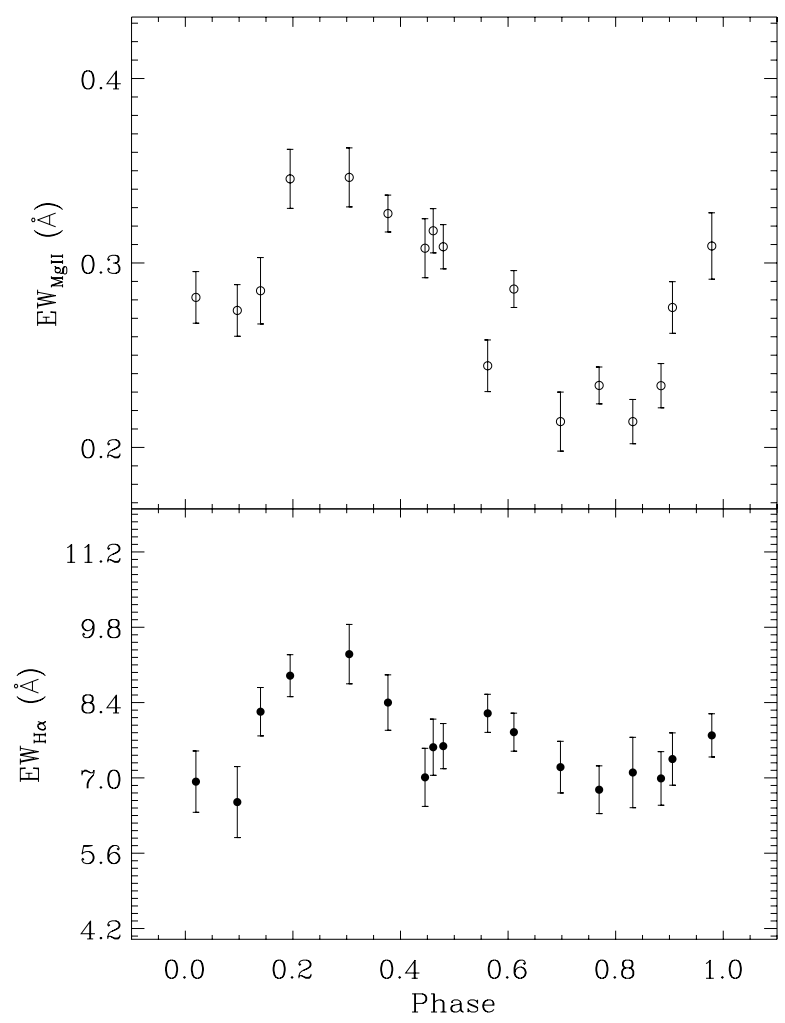

Fig. 4. Equivalent widths of MgII $\lambda 4481 \AA$ (upper panel) and $\mathrm{H}_{\alpha}$ (lower panel) lines as a function of the orbital phase.

total light should be about $0.8 \%$, which cannot detected spectroscopically with the current instrumentation.

\section{Discussion and conclusions}

The asymmetric minima and the brightness dip between the secondary and primary eclipses observed by Hall \& Hubbard (1971) and Piotrowski et al. (1974) have been explained in terms of circumstellar dust clouds. However, Martynov \& Lavrov (1972) and recently Khaliullin \& Khaliullina (2006) do not find any evident anomaly in the light curves of the system. Therefore, the idea of the presence of circumstellar dust clouds has been rejected by those authors.

With the aim of investigating this point, by means of spectroscopic tools, we have measured $E W$ of some strong lines in the spectrum of HS Her. In Fig. 4 we plot the $E W$ s of the MgII $\lambda 4481 \AA$ and $\mathrm{H}_{\alpha}$ lines of the more massive primary star, as a function of the orbital phase. A smooth modulation of both $E W \mathrm{~s}$ with a quasi-sinusoidal shape is visible. The $E W$ values are larger around the first quadrature $(\phi=0.25)$ and the minimum is reached near the second quadrature.

These observations could be explained as the effect of an extra-absorption produced by circumbinary matter with a varying optical depth along the line of sight at different phases (inhomogeneous structure). In this hypothesis, most of the matter should be concentrated in the first quarter, in contrast with the suggestion of Hall \& Hubbard (1971). Since the more massive star is well inside its corresponding Roche lobe, mass exchange between the components may be excluded. Therefore, we suggest that the circumbinary matter in this young system could be the remnant of the cloud that originated it. The presence of circumstellar or circumbinary matter in young stars is very common (e.g., Valenti et al. 2000; Klochkova 2001; 

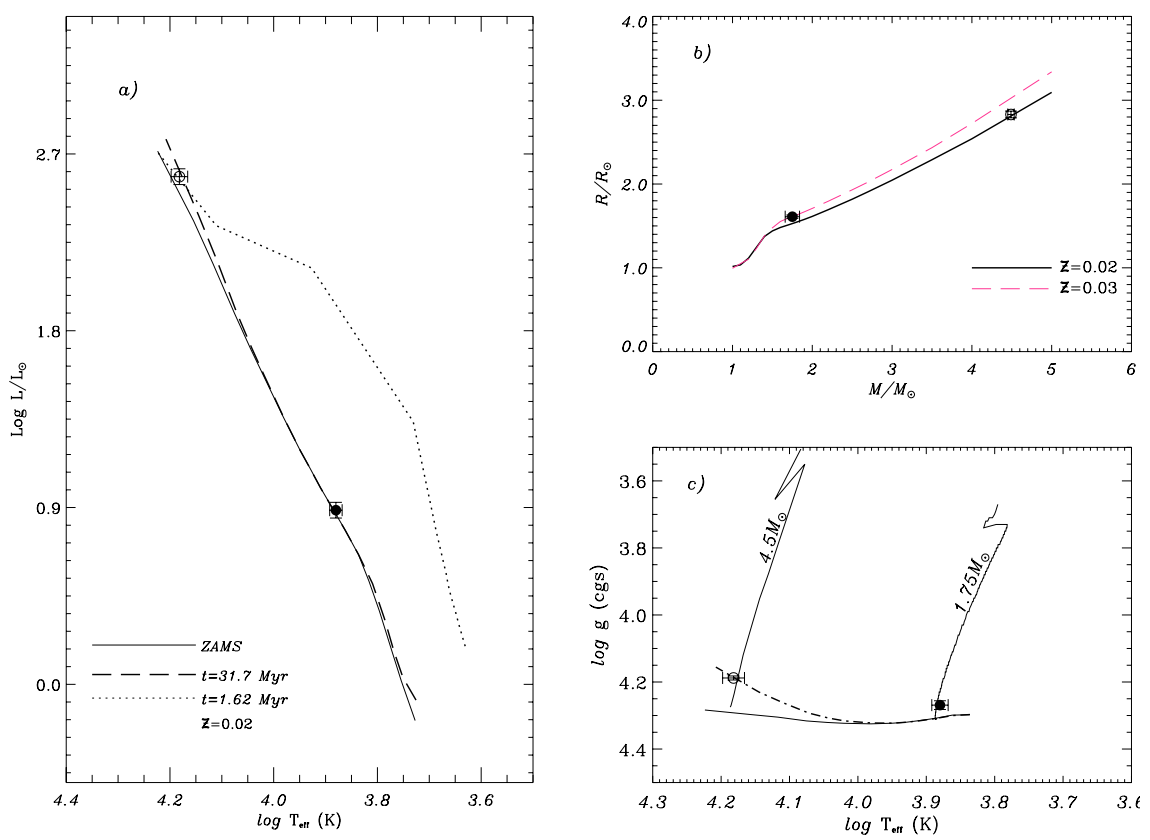

Fig. 5. a) Location of the primary and secondary stars in the theoretical H-R diagram. Dotted and dashed lines correspond to the pre-mainsequence stars with an age of 1.6 Myr and main-sequence stars with an age of $32 \mathrm{Myr}$. The solid line indicates the ZAMS. b) Comparison of theoretical models (Siess et al. 2000) with the primary and secondary stars of HS Her in the mass-radius plane. c) Comparison of theoretical models and the primary and secondary of HS Her in the $\log g-\log T_{\text {eff }}$ plane. The observed values of the components are indicated with error bars. The evolutionary tracks for 4.5 and $1.75 M_{\odot}$, taken from Siess et al. (2000), are also plotted.

Duvert et al. 1998). Such disks are intimately related to the star formation process. Angular momentum conservation during the initial contraction of a proto-stellar cloud eventually leads to a central pre-main sequence star surrounded by a disk. As angular momentum is transported outward through the disk, material must move from the disk, either onto the star or into a wind (see, e.g., Feigelson \& Montmerle 1999). Theoretical studies on star formation suggest that a substantial portion of a star's final mass is accreted through such a disk.

The optical spectra presented here are inadequate to reveal the structure of the material. Near ultraviolet and infrared spectra may reveal the presence of circumstellar absorption by neutral metals.

A number of independent studies indicate that HS Her is a young stellar system. These considerations are based on the locations of the component stars on the luminosity-temperature or gravity-age diagrams, and on the presence of a circumstellar cloud (Hall \& Hubbard 1971).

The location of the two components of HS Her on the HR and related diagrams confirms the very young age of the system, which is in agreement with the possible detection of primordial circumstellar matter indicated by the varying $\mathrm{Mg}$ II and $\mathrm{H}_{\alpha}$ absorptions.

Acknowledgements. We thank Prof. G. Strazzulla, director of the Catania Astrophysical Observatory, for his warm hospitality and allowance of telescope time for the observations. In addition, ÖÇ is grateful to all the people working at the Catania Astrophysical Observatory for creating a stimulating and enjoyable atmosphere and, in particular, to the technical staff of the OAC, P. Bruno, G. Carbonaro, A. Distefano, M. Miraglia, A. Miccichè, and G. Occhipinti, for the valuable support in carrying out the observations. The authors also acknowledge the support of the The Scientific and Technological Research Council of Turkey through its TBAG 105 T276 project. This research has been also partially supported by INAF and Italian MIUR. We thank J. Southworth, the referee, for his numerous constructive remarks and suggestions, particularly for evaluating the errors of the physical quantities. This research has been made use of the ADS-CDS databases, operated at the CDS, Strasbourg, France.

\section{References}

Bastian, U. 1993, AN, 314, 39

Bozkurt, Z., \& Değirmenci, O. L. D. 2006, Ap\&SS, 304, 29

Cesco, C., \& Shade, J. 1945, ApJ 101, 114

Chamblis, C. R. 1992, PASP, 104, 663

Colak, T., \& Müyesseroglu, Z. 2005, IBVS 5619

Demircan, O. 2000, in NATO Science Ser. C544, Variable Stars as Essential Astrophysical Tools, ed. C. İbanoğlu (Dordrech: Kluwer), 615

de Jager, C., \& Nieuwenhuijzen, H. 1987, AA, 177, 217

Drilling, J., \& Landolt, A. U. 2006, in Allen's Astrophysical Quantities, ed. A. N. Cox (New York: Springer Verlag), 381

Duvert, G., Dutrey, A., Guilloteau, S., et al. 1998, A\&A, 332, 867

Eggleton, P. 1971, MNRAS, 151, 351

Etzel, B. P., \& Olson, E. C. 1993, AJ, 106, 1200

Feigelson, E. D., \& Montmerle, T. 1999, ARA\&A, 37, 363

Frasca, A., Guillout, P., Marilli, E., et al. 2006, A\&A, 454, 301

Hall, D. S. 1971, BAAS, 3, 12

Hall, D. S., \& Hubbard, G. S. 1971, PASP, 83, 459

Hernández, J., Calvet, N., Briceño, C., Hartmann, L., \& Berlind, P. 2004, AJ, 127,1682

Hovhannessian, R. Kh. 2004, Astrophys., 47, 499

Jacchia, L. 1940, Harvard Bull., 912, 20

Khaliullin, Kh. F., \& Khaliullina, A. I. 2006, Astron. Rep., 50, 5

Martynov, D. Ya. 1940, Bull. Astr. Obs., V. P Engel'garta, 18, 38

Nordstroem, B., Mayor, M., Holmberg, et al. 2004, A\&A, 418, 989

Queloz, D., Allain, S., Mermilliod, J.-C., Bouvier, J., \& Mayor, M. 1998, A\&A, 335,183

Piotrowski, S. L., Rucinski, S. M., \& Semeniuk, I. 1974, Acta Astron., 24, 389

Royer, F., Grenier, S., Baylac, M., Gomez, A. E., \& Zorec, J. 2004, A\&A, 393, 897

Siess, L., Dufour, E., \& Forestini, M. 2000, A\&A, 358, 593

Southworth, J., Maxted, P. F. L., \& Samlley, B. 2005a, A\&A, 429, 645

Todoran, I., \& Agerer, F. 2002, AN, 315, 349

Oja, T. 1991, A\&AS, 71561

Valenti, J. A., Johns-Krull, C. M., \& Linsky, J. L. 2000, ApJS, 129, 399

Vitrichenko, A. E., Klochkova, V. G., \& Tsymbal, V. V. 2006, Astrofizika, 49, 111

Vitrichenko, A. E., \& Klochkova, V. G. 2001, Astron. Lett., 27, 328

Wolf, M., Harmanec, P., Diethelm, R., et al. 2002, A\&A, 383, 533 